\title{
Dentin Matrix Alteration
}

National Cancer Institute

\section{Source}

National Cancer Institute. Dentin Matrix Alteration. NCI Thesaurus. Code C139143.

A term that refers to alterations in the dentin matrix. Causes include dentinogenesis imperfecta and hypophosphatemic rickets. 